\title{
A Retrospective, Cohort-Based Survey of Patients Using Twice-Daily Indinavir + Ritonavir Combinations: Pharmacokinetics, Safety, and Efficacy
}

\author{
*David M. Burger, *Patricia W. H. Hugen, *Rob E. Aarnoutse, †Jeanne P. Dieleman, \\ $\ddagger$ Jan M. Prins, $\neq$ Tom van der Poll, §Jacob H. ten Veen, "Jan W. Mulder, "Pieter L. Meenhorst, \\ TWillem L. Blok, $\ddagger$ Jan T. M. van der Meer, \$Peter Reiss, and $\ddagger \#$ Joep M. A. Lange \\ * Department of Clinical Pharmacy, University Medical Centre Nijmegen; tDeparment of Intemal Medicine, University Hospital \\ Rotterdam; tDepartment of Infectious Diseases, Tropical Medicine and AIDS, Academic Medical Center, Amsterdam; \\ $\S$ Department of Internal Medicine, OLVG/Prinsengracht Hospital, Amsterdam; lDepartment of Internal Medicine, Slotervaart \\ Hospital, Amsterdam; IDepartment of Internal Medicine, Walcheren Hospital, Vlissingen; and \#National AlDS Therapy \\ Evaluation Center, Amsterdam, The Netherlands
}

\begin{abstract}
Objective: To describe the pharmacokinetics, safety, and efficacy of twice-daily indinavir + ritonavir regimens

Design: A cohort-based survey of HIV-infected patients who either used indinavir $800 \mathrm{mg}+$ ritonavir $100 \mathrm{mg}$ twice daily or indinavir $400 \mathrm{mg}$ + ritonavir $400 \mathrm{mg}$ twice daily.

Methods: Data were extracted from a database of samples sent to our laboratory for measurement of indinavir + ritonavir plasma concentrations. Patient characteristics, safety, and efficacy measurements were collected by retrospective chart review.

Results: 100 Patients using $800 \mathrm{mg}$ indinavir $+100 \mathrm{mg}$ ritonavir twice daily and 32 patients using $400-\mathrm{mg}$ indinavir $+400-\mathrm{mg}$ ritonavir twice dally were eligible. Median peak and trough concentrations of indinavir were 6.8 and $0.77 \mathrm{mg} / \mathrm{L}$ in the $800 / 100$ group and 2.6 and $0.45 \mathrm{mg} / \mathrm{L}$ in the $400 / 400$ group. The most frequently found side effects were nausea and vomiting, which occurred in $22.1 \%$ and $34.9 \%$ of the patients in the $800 / 100$ and the $400 / 400$ groups, respectively. Viral load data were analyzed for patients who switched from $800 \mathrm{mg}$ indinavir three times daily to one of the indinavir + ritonavir twice daily regimens. At the time of switch $63 \%$ (800/100 group) and $60 \%$ (400/400 group) had an undetectable viral load and this increased to $77 \%$ and $70 \%$, respectively, during follow-up. Patients who switched to the $400 / 400$ group discontinued treatment more frequently than patients who switched to the $800 / 100$ group $(70 \%$ vs. $26 \%, p=.008)$.

Conclusions: Indinavir + ritonavir regimens show improved pharmacokinetic properties, allowing twice-daily dosing with food. Clinical data suggest that safety and efficacy is at least as good as with indinavir three-times-daily regimens without ritonavir. Prospective, comparative trials are needed to properly assess the role in HIV therapy of these twice-daily indinavir + ritonavir regimens.
\end{abstract}

Key Words: Indinavir-Ritonavir-Pharmacokinetics-Efficacy-Safety,

Address correspondence and reprint requests to David $M$. Burger, Department of Clinical Pharmacy, 533KF University Medical Centre Nijmegen, P,O. Box 9101, $6500 \mathrm{HB}$ Nijmegen, The Netherlands: e-mail: D.Burger@klinfarm.azn.nl

Manuscript received August 14, 2000; accepted November 7 , 2000 .
Indinavir is one of the most widely used protease inhibitors. The popularity of this drug can be explained by its well-documented clinical efficacy, which has been reported to last at least 3 years (1), its relatively benign toxicity profile $(2,3)$, and its mild (relative to that of ritonavir) drug-drug interaction profile (4). 
In contrast to these favorable characteristics, however, use of indinavir has also some disadvantages. The drug should be taken with a low-fat, low-calorie meal or on an empty stomach, which means 1 hour before or 2 hours after a meal. Because indinavir is dosed three times daily, patients must deal with arranging meals and drug intake during a large part of the day. From a pharmacokinetic perspective, indinavir has also some unfavorable characteristics. Due to good absorption, especially when indinavir is ingested on an empty stomach, the plasma concentration increases rapidly, leading to high concentrations that may be related to nephrologic and urologic complications $(5,6)$. Furthermore, due to extensive cytochrome P450-mediated metabolism, indinavir plasma concentrations decrease rapidly, resulting in trough concentrations that are only 2 to 4 times higher than the $95 \%\left(\mathrm{IC}_{95}\right)$ inhibitory concentration. Interpatient and intrapatient variability may result in trough concentrations that are even closer to the $\mathrm{IC}_{95}$, which has been associated with suboptimal viral suppression (7-9). The small difference between indinavir trough concentration and the $\mathrm{IC}_{95}$ necessitates strict adherence to the every-8-hours regimen. Intervals of $>8$ hours between two doses and/or neglecting food requirements will result in exposure to suboptimal drug concentrations [i.e., $<0.10 \mathrm{mg} / \mathrm{L}(7,8)$ ], which may be associated with the emergence of drug resistance and, eventually, loss of antiviral effect.

If it were possible to improve the pharmacokinetic profile of indinavir while maintaining its antiviral potency and tolerability, this would be a welcome extension of today's antiretroviral therapeutic options. Combining indinavir with ritonavir is one way to improve the pharmacokinetic profile of indinavir (10). Ritonavir inhibits cytochrome P450-mediated metabolism of indinavir (and other protease inhibitors), which makes it possible to administer indinavir twice daily with or without food $(11,12)$. Healthy volunteer studies have shown that there are two different dosing strategies to combine indinavir with ritonavir: 800-mg indinavir + low-dose (e.g., 100$200 \mathrm{mg}$ ) ritonavir (12) or $400 \mathrm{mg}$ (1.1) of both protease inhibitors. Although these regimens are becoming increasingly popular in clinical practice, no systematic review of the pharmacokinetics, safety, and efficacy in HIV-infected patients has been available so far. In this study, we have investigated the pharmacokinetics, safety, and efficacy of both twice-daily indinavir + ritonavir combinations.

\section{METHODS}

\section{Patient Selection}

All physicians in the Netherlands who treat HIV-infected patients were offered the possibility of sending samples for drug concentration monitoring. Physicians were instructed to record the following information on the sample application form: reason for requesting drug concentration measurement (suspicion of drug interaction, suboptimal therapy, toxicity or noncompliance, routine control), date, dosage of antiretroviral drugs, time of blood sampling, time of last drug ingestion, and use of concomitant medications.

Information on the sample application forms was entered in a Microsoft Access 2.0 database (Microsoft, Redmond, WA, U.S.A.) between spring 1997 and July 1999. At the time of analysis, July 1999, the database contained data on $>1,000$ patients who were using indinavir as part of their antiretroviral regimen. For the purpose of this study, data were extracted from the database for patients who had been prescribed both indinavir and ritonavir as part of their antiretroviral regimen.

\section{Drug Concentration Measurement and Pharmacokinetic Analysis}

Indinavir plasma concentrations were measured according to the methods described elsewhere $(13,14)$. Ritonavir plasma concentrations were measured by the method of Hugen et al. (14). Individual drug concentrations at each specific sampling time postingestion were entered into a pharmacokinetic curve-fitting program (Kinfit, Mediware, J.H. Proost, Groningen, The Netherlands) (15). The appropriate pharmacokinetic model was selected by using Akaike's information criterion and the average population pharmacokinetic curve was drawn.

\section{Safety and Efficacy Measurements}

A brief questionnaire was sent to various hospitals to collect information on previous use of antiretroviral drugs and the reason to switch to an indinavir + ritonavir combination. Data on viral load, the occurrence of side effects, and lipid concentration measurements before and after switching to an indinavir + ritonavir combination were also requested. Physicians who had submitted samples for measuring indinavir concentrations were asked to complete these questionnaires.

\section{RESULTS}

\section{Pharmacokinetic Data}

In all, 132 patients were identified, 100 of whom used indinavir $800 \mathrm{mg}+$ low-dose ritonavir $(100 \mathrm{mg})$ twice daily (800/100 group) and 32 who used both drugs in a dose of $400 \mathrm{mg}$ twice daily ( $400 / 400$ group). A total 225 samples were analyzed: 177 from the $800 / 100$ group; 48 from the 400/400 group. Samples were taken at random times postingestion with no specific cluster of timepoints, except a relatively high number of trough samples. After entering the individual plasma concentrations of indinavir and ritonavir into the Kinfit program, pharmacokinetic parameters were calculated (Table 1). In all cases, a two-compartment model with a lag-time best fit the data. The fitted average pharmacokinetic curves of indinavir and ritonavir in both dosing regimens are shown in Figures 1 and 2. Analysis of the trough concentrations of indinavir and ritonavir in patients using 
TABLE 1. Pharmacokinetic paramaters of indinavir and ritonavir as calculated by the Kinfit program

\begin{tabular}{|c|c|c|c|c|}
\hline \multirow{2}{*}{$\frac{\text { Regimen }}{\text { Drug }}$} & \multicolumn{2}{|c|}{$800 / 100$} & \multicolumn{2}{|c|}{$400 / 400$} \\
\hline & Indinavir & Ritonavir & Indinavir & Ritonavir \\
\hline $\mathrm{C}_{\max }(\mathrm{mg} / \mathrm{L})$ & 8.9 & 2.2 & 3.3 & 9.1 \\
\hline $\mathrm{T}_{\max }(\mathrm{hr})$ & 1.4 & 3.0 & 3.4 & 3.3 \\
\hline $\mathrm{AUC}_{0-12 \mathrm{hr}}(\mathrm{mg} / \mathrm{L} \times \mathrm{hr})$ & 46.6 & 17.4 & 21.0 & 57.2 \\
\hline $\mathrm{C}_{\min }(\mathrm{mg} / \mathrm{L})$ & 1.3 & 0.53 & 0.31 & 1.5 \\
\hline $\mathrm{CL} / \mathrm{F}(\mathrm{L} / \mathrm{hr})$ & 16.3 & 5.3 & 17.9 & 6.6 \\
\hline $\mathrm{Vd} / \mathrm{F}(\mathrm{L})$ & 80.7 & 24.4 & 44.3 & 16.1 \\
\hline
\end{tabular}

$\mathrm{C}_{\max }$, maximum concentration; $\mathrm{T}_{\max }$, time of maximum concentration; CL/F; clearance; $\mathrm{Vd} / \mathrm{F}$, volume of distribution.

$800 / 100$ combination revealed a remarkably strong correlation between these two parameters. The fitted correlation has the following equation: [indinavir trough concentration in $\mathrm{mg} / \mathrm{L}$ ] $=2.01 \times$ [ritonavir trough concentration in $\mathrm{mg} / \mathrm{L}]+0.07 ; \mathrm{r}=0.89 ; p<.01$. The number of trough samples in the $400 / 400$ group was too small to repeat this analysis in this group of patients.

Several patients who suffered from toxicity while using the $800 / 100$ regimen had a dose modification to 600 $\mathrm{mg}$ or $400-\mathrm{mg}$ indinavir twice daily $+100-\mathrm{mg}$ ritonavir twice daily. The peak and trough drug concentrations that were measured while these patients followed the modified regimen are listed in Table 2. In this subgroup of selected patients, the modified drug combination regimen resulted in drug concentrations that were comparable with those observed on average in the $800 / 100$ group.

\section{Safety and Efficacy Data}

In all, 132 questionnaires were sent to participating physicians. The response rate to the questionnaire was $72 \%$ in the $400 / 400$ group and $77 \%$ in the $800 / 100$ group. Data for the $800 / 100$ group and the $400 / 400$ group are presented separately.

\section{0/100 Group}

More than half of the patients (56\%) had used 800-mg indinavir three times daily before starting $800 / 100$ twice daily. Other regimens included combinations without indinavir $(17 \%), 1200-\mathrm{mg}$ indinavir twice daily $(12 \%)$, no therapy (10\%), or the $400 / 400$ indinavir + ritonavir combination (3\%). The reasons to start $800 / 100$ twice daily were: a less complex regimen (26\%), a low indinavir plasma level (26\%), a high viral load (12\%), side effects $(10 \%)$, drug interactions $(4 \%)$ or not specified $(22 \%)$.

Side effects that were reported in more than $4 \%$ are listed in Table 3. Nausea and vomiting were the most frequently reported side effects, followed by nephrotoxicity (renal stones, hematuria, crystalluria), lipodystrophy syndrome, skin reactions, and hyperbilirubinemia. Analysis of plasma cholesterol concentrations measured before and after starting $800 / 100$ showed that the median cholesterol concentration increased from 5.2 (interquartile ratio $[\mathrm{IQR}], 4.5-6.2) \mathrm{mmol} / \mathrm{L}$ to 5.7 (IQR, 4.9-7.4) $\mathrm{mmol} / \mathrm{L}$. Median triglyceride concentrations increased from 2.5 (IQR, 1.4-4.0) $\mathrm{mmol} / \mathrm{L}$ to 3.1 (IQR, 1.9-4.4) $\mathrm{mmol} / \mathrm{L}$. Most physicians noted on the questionnaire that they did not know whether the patient was fasting, so these data must be considered as nonfasting results.

Because most patients had been using 800 -mg indinavir three times daily before switching to $800 / 100$, and the other patients represent a rather heterogenous group (treatment-naive and nonnaive; indinavir-experienced and nonexperienced, and the like), it was decided to perform the viral load analysis only in those 43 patients who switched from 800-mg indinavir three times daily to the $800 / 100$ regimen. The median follow-up of these patients on $800 / 100$ was 5 months (range, $1-13$ months). Of the 43 patients, $11(26 \%)$ had stopped taking the indinavir + ritonavir combination at the time of analysis; in 10 of these 11 patients, side effects were the cause, while in 1 case no cause was specified.

Viral load data at baseline (i.e., before switching regimens) and at the end of follow-up were available for 41 patients (the other 2 patients had HIV-2 infection). Median viral load in these patients before the switch was $<400 \mathrm{copies} / \mathrm{ml}$, with 26 of these 41 patients $(63 \%)$ having a viral load below the limit of detection (which varied from 5 to 400 copies $/ \mathrm{ml}$, depending on the assay used in each given hospital). During follow-up, the proportion of patients with an undetectable viral load increased from $63 \%$ to $78 \%$ (intention-to-treat analysis) or $77 \%$ (ontreatment analysis). Of the 26 patients with an undetectable viral load before switch, $24(92 \%)$ also had an undetectable viral load during follow-up, whereas of the 15 patients with a detectable viral load before switching, 8 $(53 \%)$ became undetectable after the switch.

\section{0/400 Group}

The largest group of patients (44\%) had taken $800-\mathrm{mg}$ indinavir three times daily before starting the $400 / 400$ regimen. Other regimens included combinations without indinavir $(30 \%)$, no therapy $(17 \%)$ or the $800 / 100$ indinavir + ritonavir combination $(9 \%)$. The reasons to start 400/400 were: a less complex regimen (43\%), a high viral load $(26 \%)$, side effects $(17 \%)$, a low indinavir plasma level $(4 \%)$, or not specified $(9 \%)$.

Side effects that were reported in more than $4 \%$ are 


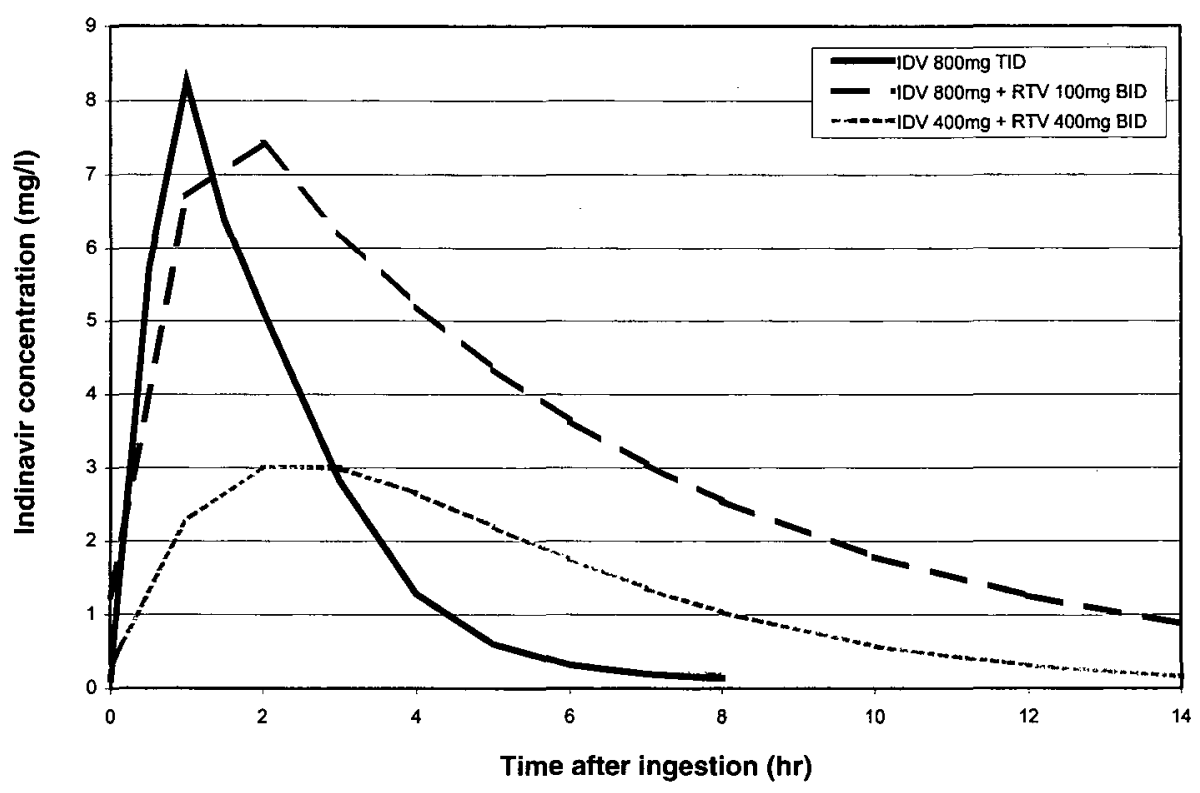

FIG. 1. Fitted pharmacokinetic curves of indinavir when administered with ritonavir.

listed in Table 3. As with the group receiving 800/100, nausea and vomiting were the most frequently reported side effects, followed by nephrotoxicity (renal stones, hematuria, crystalluria), diarrhea, and lipodystrophy. Analysis of plasma cholesterol concentrations measured before and after starting the $400 / 400$ regimen showed that the median cholesterol concentration increased from 5.2 (IQR, 4.2-5.8) $\mathrm{mmol} / \mathrm{L}$ to 5.7 (IQR, 5.4-6.6) $\mathrm{mmol} / \mathrm{L}$. Median triglyceride concentrations increased from 2.6
(IQR, 1.3-3.7) $\mathrm{mmol} / \mathrm{L}$ to 3.1 (IQR, $1.8-4.5$ ) $\mathrm{mmol} / \mathrm{L}$. Again, most physicians noted on the questionnaire that they did not know whether the patient was fasting.

Because the largest part of the patients had been taking $800-\mathrm{mg}$ indinavir three times daily before switching to $400 / 400$, and to make a comparison with the $800 / 100$ group, viral load analysis was only performed in the 10 patients who switched from $800-\mathrm{mg}$ indinavir three times daily to $400 / 400$ twice daily. The median follow-up of

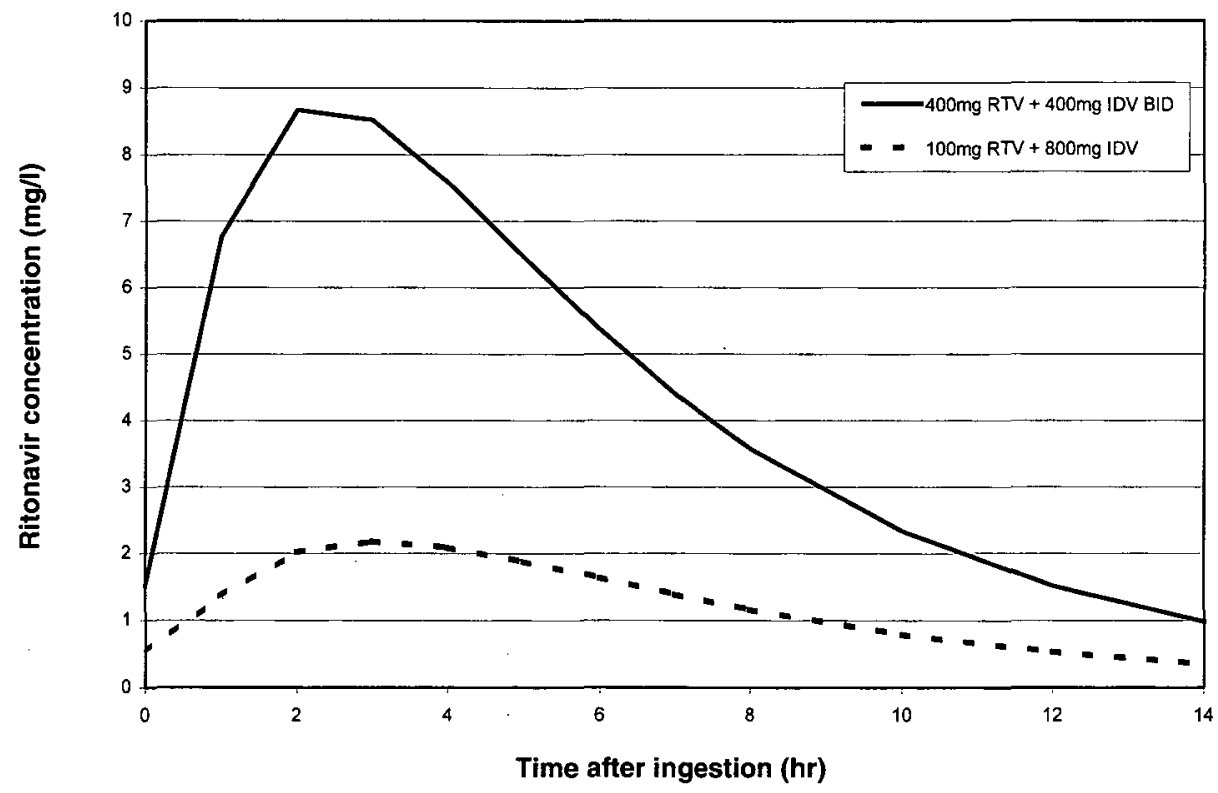

FIG. 2. Fitted pharmacokinetic curves of ritonavir when administered with indinavir. 
TABLE 2. Dosage modifications ( $800 / 100$ group only)

\begin{tabular}{lcrrr}
\hline & Dose & $N$ & $\begin{array}{c}\text { Indinavir } \\
\text { concentration } \\
(\mathrm{mg} / \mathrm{L})\end{array}$ & $\begin{array}{c}\text { Ritonavir } \\
\text { concentration } \\
(\mathrm{mg} / \mathrm{L})\end{array}$ \\
\hline Peak concentration & $400 / 100$ twice daily & 2 & $4.9 \pm 1.7$ & $2.3 \pm 1.1$ \\
& $600 / 100$ twice daily & 2 & $5.8 \pm 0.8$ & $2.2 \pm 0.2$ \\
& $800 / 100$ twice daily & 86 & $7.6 \pm 3.9$ & $2.2 \pm 1.7$ \\
Trough concentration & $400 / 100$ twice daily & 5 & $1.2 \pm 0.5$ & $0.74 \pm 0.36$ \\
& $600 / 100$ twice daily & 7 & $1.4 \pm 0.5$ & $0.64 \pm 0.18$ \\
& $800 / 100$ twice daily & 63 & $1.2 \pm 1.1$ & $0.55 \pm 0.48$ \\
\hline
\end{tabular}

these patients was 7 months (range, 1-14 months). Seven patients $(70 \%)$ had stopped taking the indinavir + ritonavir combination at the time of data analysis. Side effects were the reason given in 4 patients, 2 others stopped because of noncompliance or myocardial infarction, and no reason for discontinuation was specified for 1 patient.

The median viral load before switch was $<400 \mathrm{copies} / \mathrm{ml}$, with 6 patients $(60 \%)$ having a viral load below the limit of detection. During follow-up, the proportion of patients with an undetectable viral load increased from $60 \%$ to $70 \%$ (intention-to-treat analysis) or $100 \%$ (on-treatment analysis). All patients with an undetectable viral load before switching continued to have undetectable viral loads.

\section{DISCUSSION}

The first clinical data on combining ritonavir + indinavir were reported in 1997 at the 36th Interscience Conference on Antimicrobial Agents and Chemotherapy (ICAAC) in Toronto and published 1 year later (11). However, since then, only limited data have become available, despite widespread use of the combination in clinical practice. Until now, the pharmacokinetics of indinavir + ritonavir when both are given in a 400-mg dose twice daily have only been described in healthy volunteers $(11,12)$. Furthermore, toxicity and efficacy data of indinavir + ritonavir regimens in HIV-infected patients have only been presented in abstract form (16-19), and these studies usually involved only small numbers of patients.

In the healthy volunteer studies conducted by both Merck (12) and Abbott (11), it was demonstrated that indinavir can be given twice daily with food when combined with 100 - to $400-\mathrm{mg}$ ritonavir. The indinavir + ritonavir combination is more convenient for patients than the registered regimen that requires the intake of indinavir (without ritonavir) on an empty stomach or with a light meal every 8 hours. The healthy volunteer studies also showed that total exposure to indinavir does not differ when both drugs are given on an empty stomach or with food, so this indicates that indinavir + rito- navir represents the first dual PI combination that can be administered without food restrictions. It may be advisable, however, to take indinavir + ritonavir with food because this results in blunting of the peak indinavir concentration (see following discussion) $(11,12)$. Although it is clear from Figure 1 that both twice daily regimens of indinavir + ritonavir result in improved pharmacokinetics of indinavir compared with the 800$\mathrm{mg}$ three-times-daily regimen of indinavir without ritonavir, the two twice-daily regimens are not interchangeable $(11,12)$. The $800 / 100$ regimen leads to similar peak concentrations of indinavir as is the situation with the 800-mg three-times-daily regimen of indinavir without ritonavir, although trough concentrations are approximately tenfold higher. These data are in agreement with those from the healthy volunteer study (12) and the 6 patients reported by Van Heeswijk et al. (20). The indinavir peak concentrations in the 400/400 regimen are substantially lower than with either of the two other regimens, whereas trough concentrations fall somewhere between those obtained with the 800-mg three times daily and the $800 / 100$ twice daily regimens. These data are in accordance with the healthy volunteer data (11). The implications of these differences have been the subject of much debate at recent international conferences. Most attention has focused on the differences in peak indinavir concentrations between the two twice-daily regimens because the development of nephrotoxicity, caused by precipitation of indinavir base in the kidneys, may be related to the height of the indinavir concentration in plasma (5). Given the values of peak indinavir concentration in this study, it can be expected that the incidence of nephrotoxicity in the $800 / 100$ twice-daily regimen will equal the incidence in patients using indinavir $800 \mathrm{mg}$ three times daily without ritonavir, whereas incidence in the $400 / 400$ regimen will probably be lower. This is in agreement with the reported incidences of nephrotoxicity shown in Table 3. Less attention has been paid to possible differences in indinavir trough concentrations between the various regimens. Our data show that indinavir trough concentrations in the $800 / 100$ twice daily regimen are substantially higher than observed in either of the

TABLE 3. Side effects

\begin{tabular}{lcc}
\hline \multicolumn{1}{c}{ Regimen } & $800 / 100$ & $400 / 400$ \\
\hline$N$ & 77 & 23 \\
Nausea, vomiting & $22.1 \%$ & $34.9 \%$ \\
Renal stones, hematuria, crystalluria & $14.3 \%$ & $8.7 \%$ \\
Lipodystrophy & $13.0 \%$ & $4.3 \%$ \\
Hyperbilirubinemia & $11.7 \%$ & - \\
Diarrhea & $5.2 \%$ & $4.3 \%$ \\
Skin reaction & $13.0 \%$ & - \\
\hline
\end{tabular}


other regimens (Table 1, Fig. 2). This may allow the $800 / 100$ twice-daily regimen to suppress HIV with moderately decreased susceptibility to indinavir (i.e., a 4-fold to 10-fold increase in $\mathrm{IC}_{95}$ ) because of the 10-fold higher indinavir trough concentrations. Trough concentrations of indinavir after intake of the 400/400 regimen may not be sufficiently high enough to overcome such an increase in $\mathrm{IC}_{95}$. Because of the almost $100 \%$ cross-resistance between indinavir and ritonavir (21), it seems unlikely that ritonavir in the 400/400 combination will have much virologic effect on virus with decreased susceptibility to either drug.

The interpretation of the reported side effects is complicated. First, this study was not randomized and patients may not have been similar while starting one of the twice-daily regimens. Second, the data collection may be unreliable because physicians may remember the most recent or the most impressive side effects most clearly. Third, some side effects may result from incorrect use of the drugs. For example, the first patients who started on the $800 / 100$ regimen did so by taking the drugs on an empty stomach because no information on influence of food on this regimen was known at that time. This may have led to higher peak concentrations of indinavir than after eating because food blunts the peak concentration as a result of delayed absorption $(11,12)$. Another problem in the evaluation of side effects in this study may be that such side effects were already present with the previous regimen (e.g., lipodystrophy syndrome) and are not likely to be specifically related to the indinavir + ritonavir regimen. Therefore, no definitive conclusions can be drawn concerning any differences in toxicities between the two twice-daily regimens.

Given the uncertainty of reporting side effects in this study, the following observations ought be noted. Nausea is a well known side effect of both indinavir and ritonavir, so it is not surprising that this side effect was reported most frequently in the questionnaires. The higher incidence of nephrotoxicity (defined as kidney stones, hematuria, or crystalluria) in the $800 / 100$ regimen when compared with the $400 / 400$ regimen is in agreement with the already discussed differences in indinavir peak concentrations. It must also be noted, however, that the incidence of nephrotoxicity in the $400 / 400$ regimen is not zero in this study, in contrast to the data reported by Workman et al. (22). Padberg et al. (23) recently reported a $12 \%$ incidence of elevated serum creatinine levels in patients using 400-mg indinavir $+400-\mathrm{mg}$ ritonavir twice daily. A $32 \%$ incidence in nephrotoxicity was reported by O'Brien et al. (16), but they were using a combination of $800-\mathrm{mg}$ indinavir $+200-\mathrm{mg}$ ritonavir, which leads to higher peak concentrations of indinavir than with $800 / 100$ (12). Furthermore, their patients were suffering from an extremely hot summer in Texas at the time the data were being collected (W. O'Brien, oral communication, April 1999).

Recent data from Youle et al. (24) suggest that, when combined with indinavir, higher ritonavir doses lead to a more pronounced risk of lipid abnormalities than with lower ritonavir doses. Given the uncertain long-term effects of increased lipid concentrations, minimal changes in lipid concentrations are desirable, which suggests a preference for the $800 / 100$ regimen over the $400 / 400$ regimen. Our data do not confirm these observations of Youle et al. Nonstandardized (i.e., nonfasting) measurements of lipids in our study and small numbers in the various regimens in Youle's study (there were only 2 patients in their 800/100 group) may explain the difference in the findings.

This study is the first to report on the virologic efficacy of the $800 / 100$ regimen. In the subset of patients who switched from 800-mg indinavir three times daily to the 800/100 regimen, almost all patients who had an undetectable viral load remained undetectable, whereas a significant proportion of patients with a detectable viral load became undetectable. This indicates that the antiviral activity of the $800 / 100$ combination is at least similar to regimens containing $800-\mathrm{mg}$ indinavir three times daily. Although not specified in the questionnaire, most physicians reported that nucleosides were not changed when adding low-dose ritonavir. Approximately $25 \%$ of the patients in the $800 / 100$ group discontinued this regimen, showing that tolerability issues may compromise the antiviral activity. Most physicians were not aware that dose modification to $600 / 100$ or even $400 / 100$ is possible. All patients who had a dose modification because of toxicity from the $800 / 100$ regimen had adequate trough indinavir concentrations (Table 2) and demonstrated sustained virologic response. Patients suffering from toxicity while using the $800 / 100$ regimen are likely to represent a subpopulation of patients with greater than average increased exposure to indinavir, and they can benefit from dosage modifications. Because there were only 10 patients who switched from an $800-\mathrm{mg}$ indinavir three times daily regimen to the $400 / 400$ twice-daily regimen, it is difficult to compare the virologic potency and tolerability of this combination with the $800 / 100$ twice-daily regimen. Patients who switched to the $400 /$ 400 group discontinued treatment more frequently than patients who switched to the $800 / 100$ group (70\% vs. $26 \%, p=.008$; using the two-sample proportion test). We have no experience in modifying the dose in the $400 / 400$ regimen. The observed intolerance may be partly due to the use of the liquid formulation of ritona- 
vir, because capsules were not available during most of the time during which this survey was conducted. The same phenomenon was observed in the study of Rockstroh et al. (19). Whether the discontinuation rate will be lower using ritonavir capsules instead of a liquid formulation remains to be established. All patients who were able to tolerate the $400 / 400$ regimen had a good virologic response ( 3 of 3 patients with an undetectable viral load in the on-treatment analysis), which is in agreement with the data from Rockstroh et al. (19) and Workman et al. (25).

In conclusion, both the $800 / 100$ and the $400 / 400$ twice-daily regimens showed improved pharmacokinetic properties when compared with regimens supplying 800$\mathrm{mg}$ indinavir three times daily without ritonavir. The virologic efficacy of both regimens appears to be at least similar to what is observed with $800-\mathrm{mg}$ indinavir three times daily. The $800 / 100$ regimen had a lower discontinuation rate than the $400 / 400$ regimen. Prospective, comparative trials are needed to properly assess the role in HIV therapy of these twice-daily indinavir + ritonavir regimens.

Acknowledgments: Michel Broekman, Joke Damsma, Jackie Droste, Marga de Graaff, Elly Kimenai, Mikel Maas, and Corrien Verwey are kindly acknowledged for analyzing plasma samples. In addition to the coauthors, the following physicians participated in data collection: M. E. van de Ende, I. C. Gyssens, S. de Marie, C. Richter, R. van Leusen, N. A. Foudraine, S. J. M. Schieveld, M. Hillebrand, F. Wit, F. P. Kroon, and F. J. B. Nellen. Abbott BV, Hoofddorp, The Netherlands (E. van Leeuwen) is acknowledged for financial support of the ritonavir analyses.

\section{REFERENCES}

1. Gulick R, Mellors J, Havlir D, et al. Treatment with indinavir, zidovudine and lamivudine: three year follow-up [abstract 388]. 6th Conference on Retroviruses and Opportunistic Infections, Chicago, Illinois, U.S.A., January 31-February 4, 1999.

2. Notermans DW, Leeuwen van R, Lange JMA. Treatment of HIV infection. Tolerability of commonly used antiretroviral agents. Drug Safety 1996;15:176-87.

3. Schooley RT. Longer-term immunologic effects and side effects of successful antiretroviral therapy. Clin Infect Dis 1999;29:12-18.

4. Burger DM, Hoetelmans RMW, Koopmans PP, et al. Clinically relevant drug interactions with antiretroviral agents. Antiviral Ther 1997;2:149-65.

5. Dieleman JP, Gyssens IC, van der Ende ME, et al. Urological complaints in relation to indinavir plasma concentrations in HIVinfected patients. AIDS 1999;13:473-8.

6. Burger DM, Swelsen W, Hugen PWH, et al., on behalf of the CHEESE study group. Indinavir (IDV) pharmacokinetics are related to efficacy and toxicity [abstract 826]. 7th European Conference on Clinical Aspects and Treatment of HIV Infection, Lisbon, Portugal, October 23-27, 1999.

7. Burger DM, Hoetelmans RMW, Hugen PWH, et al. Low plasma concentrations of indinavir are related to virological treatment failure in HIV-1 infected patients on indinavir-containing triple therapy. Antiviral Ther 1998;3:215-20.

8. Harris M, Durakovic C, Rae S, et al. A pilot study of nevirapine, indinavir and lamivudine among patients with advanced human immunodeficiency virus disease who have had failure of combination nucleoside therapy. $J$ Infect Dis 1998;177:1514-20.

9. Stein DS, Fish DG, Bilello JA, et al. A 24-week open-label phase I/II evaluation of the HIV-protease inhibitor MK-39 (indinavir). AIDS 1996;10:485-92.

10. Kempf DJ, Marsh KC, Kumar G, et al. Pharmacokinetic enhancement of inhibitors of the human immunodeficiency virus protease by coadministration with ritonavir. Antimicrob Agents Chemother 1997;41:654-60.

11. Hsu A, Granneman GR, Cao G, et al. Pharmacokinetic interaction between ritonavir and indinavir in healthy volunteers. Antimicrob Agents Chemother 1998;42:2784-91.

12. Saah A, Winchell G, Seniuk M, et al. Multiple-dose pharmacokinetics and tolerability of indinavir-ritonavir combinations in healthy volunteers [abstract 362]. 6th Conference on Retroviruses and Opportunistic Infections, Chicago, Illinois, U.S.A., January 31-February 4, 1999.

13. Burger DM, Graaff DM, Wuis EW, et al. Determination of indinavir, an HIV-protease inhibitor, in human plasma by reversedphase high-performance liquid chromatography. J Chromatogr Biomed Appl 1997;703:235-41.

14. Hugen PWH, Verwey-van Wissen CPWGM, Burger DM, et al. Simultaneous determination of the HIV-protease inhibitors indinavir, nelfinavir, saquinavir and ritonavir in human plasma by reversed-phase high-performance liquid chromatography. $J$ Chromatogr Biomed Appl 1999;727:139-49.

15. Proost JH, Meijer DKF. MW/PHARM, an integrated software package for drug dosage regimen calculation and therapeutic drug monitoring. Compt Biol Med 1992;22:155-63.

16. O'Brien WA, Atkinson TA, Han X, et al. Combination therapy with indinavir and ritonavir in antiretroviral-experienced patients [abstract 2209]. 39th Interscience Conference on Antimicrobial Agents and Chemotherapy, San Francisco, California, U.S.A., September 26-29, 1999.

17. Johnston Roberts K, Volberding P. Adherence communication: a qualitative analysis of physician-patient dialogue. AIDS 1999;13: $1771-8$.

18. Funk $M$, Linde $R$, Wintergerst $U$, et al. Preliminary experiences with triple therapy including nelfinavir and two reverse transcriptase inhibitors in previously untreated HIV-infected children. AIDS 1999;13:1653-8.

19. Rockstroh J, Bergmann F, Wiesel W, et al. BID first-line ritonavir/ indinavir $400 / 400 \mathrm{MG}$ plus double nucleoside combination therapy [abstract 452]. 7th European Conference on Clinical Aspects and Treatment of HIV Infection, Lisbon, Portugal, October 23-27, 1999.

20. Heeswijk van RPG, Veldkamp AI, Hoetelmans RMW, et al. The steady-state pharmacokinetics of indinavir alone and in combination with a low dose of ritonavir in twice daily dosing regimens in HIV-1-infected individuals. AIDS 1999;13:F95-9.

21. Hertogs K, Bloor S, Kemp SD, et al. Phenotypic and genotypic analysis of clinical HIV-1 isolates reveals extensive proteaseinhibitor cross-resistance: a survey of over 6000 samples. AIDS 2000;14:1203-10.

22. Workman C, Whittaker W, Dyer W, et al. Combining ritonavir (RTV) \& Indinavir (IDV) decreases IDV-associated urinary \& renal adverse events (AES) [abstract 116]. 7th European Conference on Clinical Aspects and Treatment of HIV Infection, Lisbon, Portugal, October 23-27, 1999.

23. Padberg J, Fritsche L, Bergmann F, et al. Nephropathy and renal colic in patients treated with indinavir, ritonavir + indinavir or ritonavir + saquinavir. AIDS 1999;13:2173-4.

24. Youle M, Mocroft A, Johnson M, et al. Lipid profiles in patients on ritonavir/indinavir-containing salvage regimens [abstract 1290]. 39th ICAAC, San Francisco, California, U.S.A., September 26-29, 1999.

25. Workman C, Whittaker W, Dyer W, et al. Virologic \& immunologic response to combination protease inhibitor (PI) therapy with ritonavir (RTV) \& indinavir (IDV) in antiretroviral naive HIV + patients [abstract 620]. 7th European Conference on Clinical Aspects and Treatment of HIV Infection, Lisbon, Portugal, October 23-27, 1999. 